\title{
Engagement et non-engagement dans les appels à la majorité des politiciens
}

Commitment and Non-commitment in the Politicians' Appeals to the Majority

\section{Corina Andone}

Translator. Marianne Doury

\section{CpenEdition Journals}

Electronic version

URL: http://journals.openedition.org/aad/2021

DOI: 10.4000/aad.2021

ISSN: 1565-8961

Publisher

Université de Tel-Aviv

Electronic reference

Corina Andone, "Engagement et non-engagement dans les appels à la majorité des politiciens », Argumentation et Analyse du Discours [Online], 15 | 2015, Online since 15 October 2015, connection on 23 September 2019. URL : http://journals.openedition.org/aad/2021 ; DOI : 10.4000/aad.2021

This text was automatically generated on 23 September 2019.

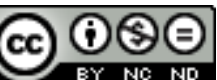

Argumentation \& analyse du discours est mis à disposition selon les termes de la licence Creative Commons Attribution - Pas d'Utilisation Commerciale - Pas de Modification 4.0 International. 


\title{
Engagement et non-engagement dans les appels à la majorité des politiciens
}

\author{
Commitment and Non-commitment in the Politicians' Appeals to the Majority
}

\author{
Corina Andone
}

Translation : Marianne Doury

\section{Introduction}

1 C'est une pratique courante, chez les hommes et femmes politiques, que d'affirmer qu'une mesure particulière doit être mise en œuvre, qu'une certaine décision doit être prise, ou qu'il faut suivre un programme donné parce que la ligne d'action proposée est susceptible d'avoir des conséquences positives [ou négatives] (« il faut baisser l'impôt sur le revenu pour que les consommateurs puissent acheter davantage de biens »). Pour convaincre leurs partenaires de débat que leur recommandation est acceptable, les politiciens font couramment appel à la majorité ( «c'est ce que la plupart des gens veulent en ces temps difficiles») (Andone 2015). Par de tels appels, les politiciens laissent entendre que leurs actes sont légitimes parce qu'ils traduisent la volonté du peuple (Anderson 1979: 721). Notamment dans le cas de controverses comportant des enjeux importants, les politiciens à l'affût de suffrages sont dans l'obligation de prôner, puis de mettre en œuvre, des options politiques qui ont la faveur de la majorité ; faute de quoi ils courent le risque de se voir reprocher un comportement antidémocratique (Cook, Barabas \& Page 2002, Reinhard, Biesenbender \& Holzinger 2014).

2 Bien qu'ils aient indéniablement pour effet d'accroittre la légitimité d'une ligne d'action, les appels à la majorité sont le plus souvent employés dans le but de réduire l'engagement du locuteur à l'égard d'une position donnée, parfois jusqu'à dénier tout engagement. En témoigne l'observation que, grâce au recours à l'appel à la majorité, les politiciens n'ont à assumer qu'une charge de la preuve limitée, voire aucune, ce qui accroît d'autant leur chance de l'emporter dans la discussion. 
3 Afin de tester cette hypothèse, cet article propose d'examiner les différents degrés d'engagement attachés aux appels à la majorité et leurs effets sur l'issue du débat. Il cherche à identifier les indicateurs et expressions qui atténuent la force avec laquelle quelque chose est affirmé. Une attention particulière sera prêtée aux indicateurs d'attitude propositionnelle (comme «je pense [que c'est ce que la majorité attend de nous]», «j'ai l'impression [que c'est ce que les gens veulent]») communément employés par les politiciens de manière à n'assumer qu'une faible charge de la preuve, voire pas de charge de la preuve du tout. Dans ce but, des exemples issus d'entretiens politiques et de débats parlementaires serviront de matériel empirique.

4 Cet article s'organise comme suit. Dans la première partie, j'aborderai la littérature qui traite des appels à la majorité, puis j'exposerai mon propre point de vue, ancré dans la pragma-dialectique, sur ces appels lorsqu'ils sont employés dans le discours politique. Dans la seconde partie, je démontrerai à partir d'exemples quels sont les engagements pris par les politiciens lorsqu'ils font appel à la majorité pour défendre une future ligne d'action. En outre, je montrerai comment ces différents engagements, ou le refus d'engagement, rejaillissent sur la charge de la preuve que doivent assumer les politiciens. Enfin, j'exposerai certains des enjeux de recherche attachés à l'étude des appels à la majorité et proposerai quelques orientations pour de futurs travaux.

\section{L'appel à la majorité}

5 Les spécialistes en sciences politiques, droit et sciences sociales, lorsqu'ils se penchent sur les appels à la majorité, soulignent souvent le rôle fondamental qu'ils jouent dans l'action politique. Ainsi que le rappelle Anderson (1979: 720), «dans tout système démocratique, la volonté de la majorité [...] est reconnue comme l'épreuve ultime d'autorité. Une politique est légitimée dans la mesure où elle reflète la volonté du peuple. » Tout en reconnaissant le rôle central des appels à la majorité, certains auteurs mettent en garde contre la conception simpliste de la démocratie qui peut y être associée, et soutiennent que le principe majoritaire ne peut être utilisé de façon exclusive, mais uniquement conjointement à d'autres arguments, ou qu'il doit être luimême étayé par des justifications additionnelles. Anderson (1979 : 721), pour sa part, avance que «sauf à être des majoritairiens absolus » éminemment dogmatiques, nous reconnaissons que considérer la volonté de la majorité comme la seule norme d'évaluation de la politique est tout à la fois paradoxal et peu satisfaisant. »

6 Malgré leur rôle central dans l'action politique, les appels à la majorité n'ont suscité que relativement peu d'attention dans les études sur l'argumentation, en comparaison avec d'autres formes argumentatives (voir Walton 1999, van Eemeren \& Houtlosser 2008, Godden 2008). Dans les différentes analyses qui en sont proposées, la majorité est invoquée comme une raison de tenir une proposition pour vraie, plutôt que comme fondement d'une action politique. Qui plus est, à quelques exceptions près (Walton 1992, van Eemeren 2010), la plupart des auteurs ont abordé les appels à la majorité comme des arguments fallacieux, autrement dit comme des arguments qui apportent des preuves discutables de la vérité de la proposition en question, mettant ainsi les appels à la majorité sur le même plan que les appels à l'opinion publique, ou argumentum ad populum ${ }^{1}$. Proposant une approche épistémique de ces derniers, Godden (2008: 104), quant à lui, observe que «l'opinion publique est en général considérée 
comme incapable de renforcer l'acceptabilité d'une proposition. De fait, les analyses standards classent les appels à l'opinion publique parmi les arguments fallacieux. »

7 De manière générale, les appels à la majorité sont caractérisés comme des arguments qui visent à justifier une affirmation en s'appuyant sur sa supposée popularité (Toulmin, Rieke \& Janik 1979 : 174), ou en faisant appel aux émotions de l'auditoire (van Eemeren \& Houtlosser 2008). D'autres auteurs, s'intéressant davantage aux argumentations destinées à justifier des croyances, entendent par «appel à la majorité » tout argument avancé en vue de fournir la preuve d'une proposition $p$ en laquelle tout le monde croit déjà (Govier 2005 : 187). Minot (1981 : 230) et Walton (1999) considèrent la forme argumentative suivante comme représentative de ces appels à l'opinion populaire: Tout le monde croit en $p$. Donc, $p$ est vrai. Walton affirme que l'on ne peut considérer que pareilles inférences offrent une preuve satisfaisante de leur conclusion; cependant, si on les considère comme révisables, elles apportent un fondement « plausible, mais provisoire » à leurs conclusions. Dans l'optique de Walton (1992 : 77), « les prémisses supplémentaires visant à étayer [...] [la conclusion] semblent avoir un statut de présomption d'arrière-plan ». Il propose un "schéma élargi» comportant deux prémisses explicites et une prémisse implicite :

P1 Tout le monde (ou tout le monde dans un groupe donné) accepte A comme une proposition vraie.

P2 Ces personnes sont en position de savoir que A est vrai, ou en tout cas,

[P2*] [Ces personnes] sont supposées avoir une raison d'accepter A.

[C] Donc, on peut accepter A comme vrai. ${ }^{2}$

8 Cette manière de reconstruire les appels à la majorité s'est vue critiquer, parce que dans cette représentation, il ressort clairement que de nouvelles preuves sont avancées en faveur de la conclusion; dès lors, on n'a plus affaire au même type d'argument (Godden 2008 : 108). Pour Godden, cette reconstruction transforme l'appel à l'opinion populaire en argument émis d'une position privilégiée ( $a$ position-to-know argument »). Il explique ainsi que «dans l'argument du premier type, le fait qu'une affirmation est acceptée par une population est utilisé comme preuve de l'acceptabilité de cette affirmation. Dans le second, le fait qu'une affirmation est admise ou non ne contribue pas en lui-même à établir l'acceptabilité de cette affirmation. On postule plutôt que certains croient en la proposition en question parce qu'elle est acceptable et qu'ils sont en possession d'éléments qui fondent cette acceptabilité » (2008: 108). Walton luimême avance, dans une autre publication, que «de nombreuses affirmations en appelant à l'opinion populaire, quand on les examine soigneusement, ne sont pas seulement des affirmations que tout le monde croit telle ou telle chose. Implicitement ou explicitement, ce sont des affirmations que tout le monde croit telle ou telle chose parce que telle ou telle chose est admise de tous $»(1999: 110)^{3}$.

Pour clarifier ce qu'est un appel à l'opinion publique dans sa " forme de base », Godden (2008: 106) en propose la reconstruction suivante, qui vise à corriger les défauts de l'approche de Walton : $p$ est largement admis dans S. Donc, $p$ est vrai. Pour Godden, cette version corrigée restitue «la structure justificative générale des appels tout en préservant les qualités [...] probatoires qui leur sont habituellement associées » (2008: 107).

10 Dans ma propre caractérisation pragma-dialectique des appels à la majorité (cf. van Eemeren \& Houtlosser 2008, van Eemeren 2010), je pars de l'hypothèse que cette façon d'argumenter, comme tout autre argument, est en soi raisonnable, mais peut devenir fallacieuse dans certaines circonstances, si certaines conditions de validité ne sont pas 
remplies. Lorsque cela se produit, en appeler à la majorité ne contribue pas à résoudre les différences d'opinion sur le fond (van Eemeren 2010). Ce traitement des appels à la majorité rend justice au fait que celui qui les emploie cherche à résoudre la différence d'opinions dans laquelle il est engagé par une discussion de fond, sans lui attribuer $a$ priori l'intention de remporter le débat à tout prix ${ }^{4}$.

11 De plus, si nous voulons être réaliste dans notre restitution des appels à la majorité, nous devons, en accord avec les spécialistes en sciences politiques, considérer que pareilles formes d'argumentation sont utilisées pour asseoir la légitimité d'une politique sans s'engager sur la vérité de la proposition politique en discussion, mais seulement sur son acceptabilité. Lorsque de tels arguments sont employés pour justifier des préconisations politiques, comme c'est le cas dans le présent article, analyser les appels à la majorité exclusivement en termes de vérité est une limitation qui n'est pas souhaitable.

Les approches existantes de cette forme d'argumentation prennent une tournure psychologique lorsqu'elles font mention des croyances, comme dans «tout le monde croit que $p »-$ formulation adoptée par la plupart des auteurs pour expliquer les appels à la majorité. L'approche pragma-dialectique de ce type d'argument peut faire l'économie de cette notion de croyance, ainsi que des outils nécessaires à sa compréhension. Elle s'en tient à évaluer l'opinion de celui qui argumente à la lumière des engagements qu'il prend, engagements identifiables par les différents actes de langage qu'il avance au cours du débat.

13 Par ailleurs, l'emploi du terme "tout le monde » ou "tout le monde dans un groupe » pour désigner la majorité ne semble pas être le meilleur choix, parce qu'il amène à considérer la majorité en termes absolus. Comme le souligne Maillat (2013 : 191), de telles formulations suggèrent qu'il y a volonté de manipulation. Dans le cas de « tout le monde ", le ressort manipulatoire consiste à affirmer qu'absolument tout le monde croit quelque chose. Lorsque la portée référentielle est limitée, comme dans «tout le monde dans un certain groupe ", il y a également de la manipulation en jeu au sens où le nombre de locuteurs qui expriment une pensée est utilisé comme argument pour la validité inhérente de cette pensée, «écartant le fait que tout le monde, c'est-à-dire tous-ceux-à-qui-j'en-ai-parlé, pourrait se tromper » (Maillat 2013 : 191). Si nous voulons considérer les appels à la majorité comme des arguments raisonnables, alors parler simplement de "majorité ", ou d'un "groupe important de personnes", rend mieux justice à la manière dont cette forme d'argumentation est employée par des locuteurs engagés dans des débats argumentés.

Pour ma part, je propose de définir les appels à la majorité comme des argumentations soutenant l'acceptabilité d'une thèse en faisant appel aux désirs, aux préférences et aux valeurs d'un grand nombre de personnes. Après tout, la démocratie est censée être mise en œuvre par des décideurs politiques attentifs au public; on peut donc s'attendre à ce qu'ils adossent chacune des propositions qu'ils avancent aux préférences de ce public en matière de politique (Cook, Barabas \& Page 2002). Cette perspective sur les appels à la majorité met en lumière que la légitimité d'une proposition politique peut être accrue par de tels appels à la majorité tant sur le plan quantitatif (on en appelle à un grand nombre de personnes) que qualitatif (on invoque les préférences des gens, c'est-à-dire ce qu'ils considèrent comme acceptable). De plus - et c'est de la plus haute pertinence pour l'objectif du présent article, il devient alors possible de considérer que les appels à la majorité viennent en soutien d'une proposition prescriptive, 
recommandant une ligne d'action, plutôt que d'une proposition descriptive, où l'on affirme que quelque chose est vrai.

\section{Engagement et non-engagement dans les appels à la majorité : des preuves par l'exemple}

Faire appel à la majorité suppose la réalisation d'un acte de langage au moyen duquel le débatteur s'engage à différents degrés sur l'acceptabilité de la proposition avancée, ainsi que sur certaines intentions (Searle 1969, Green 2007, de Brabanter \& Dendale 2008). Des auteurs comme Brandom (1994) attribuent aux engagements un statut présuppositionnel; ils entendent par là que les engagements sont évoqués de manière implicite. Cela peut se faire en exacerbant la force illocutoire des actes en question (en disant par exemple que " la majorité veut à coup sûr cela », où « à coup sûr » intensifie l'engagement du locuteur envers une ligne d'action) ou en exprimant un moindre degré d'engagement par le recours à des atténuateurs modaux comme «pourrait » («la majorité pourrait bien vouloir cela »).

La meilleure manière de comprendre, dans le discours politique, les différentes façons de modifier la force illocutoire d'un appel à la majorité consiste à prendre en compte le public, vu comme l'instance hétérogène à laquelle le discours s'adresse (Fetzer 2008 : 386). Le destinataire principal étant, dans le discours politique, constitué par le grand public, le débatteur assume des engagements qui dépassent la dyade locuteur/auditeur. Dans ce qui suit, j'étudierai dans quelle mesure les politiciens font usage de différents dispositifs de qualification visant à alléger leur charge de la preuve. Je m'attacherai à l'emploi d'appels à la majorité en soutien à une ligne d'action à venir (« il faut mettre en œuvre cette mesure »). A l'aide d'exemples, j'examinerai des cas en apparence contradictoires où des politiciens tentent de renforcer un avis (exprimé par l'énoncé de préconisation) tout en réduisant leur engagement à défendre l'acceptabilité de la ligne d'action proposée (présentée dans l'appel à la majorité).

17 Le premier exemple est extrait d'un débat en séance plénière au Parlement européen qui s'est déroulé le 9 mars 2015. Ce débat concerne la question de l'égalité entre hommes et femmes; il a été déclenché par un rapport de la Commission européenne montrant que les inégalités entre genres sont encore monnaie courante en Europe et que le niveau de violence envers les femmes est élevé. La représentante lettone Věra Jourová, membre de la Commission européenne, souligne dans son intervention l'importance de l'égalité entre les genres de la façon suivante :

Exemple (1) :

L'égalité entre les genres est un engagement de longue date pour l'UE. Les résultats d'une récente étude de l'Eurobaromètre montrent que pratiquement tous les Européens s'accordent à considérer que l'égalité entre femmes et hommes est un droit fondamental, et une large majorité de citoyens pense que traiter l'inégalité entre femmes et hommes doit être une priorité de l'UE.

Par ces remarques, Věra Jourová tente de convaincre les membres du Parlement européen qu'il est important de prendre en compte le problème de l'égalité des genres, parce que certains députés ne semblent pas vraiment voir pour quelle raison cette question devrait devenir une priorité. On peut reconstruire son argumentation de la manière suivante : 
(1) (Nous devrions instaurer comme priorité le traitement des inégalités liées au genre $)^{5}$

(1).1a Pratiquement tous les Européens s'accordent à considérer que l'égalité entre femmes et hommes est un droit fondamental.

(1).1a.1 C'est ce que montre l'Eurobaromètre.

(1).1b Une large majorité de citoyens pense que traiter l'inégalité entre femmes et hommes doit être une priorité de l'UE.

(1.)1b.1 C'est ce que montre l'Eurobaromètre. argumentation coordonnée. Dans son premier argument (1.1a), l'oratrice invoque «pratiquement tous les Européens » qui semblent considérer que l'égalité des genres est un droit fondamental. Anticipant sur la possibilité que cet argument ne suffise pas à convaincre l'auditoire de l'acceptabilité de la préconisation (1), Věra Jourová avance un autre argument (1.1b) selon lequel « une large majorité de citoyens » est d'avis que les inégalités entre les genres doivent devenir une priorité. Ces deux arguments sont employés comme éléments de preuve en faveur de la conclusion, tant sur le plan de la qualité que sur celui de la qualité. Sur le plan quantitatif, l'intervenante fait référence de façon hyperbolique dans l'argument (1).1a à « pratiquement tous les Européens », ce qui naturellement pose la question de savoir si tous les Européens ont pris part à l'Eurobaromètre. Cet argument donne le sentiment que la politicienne détient des informations factuelles incontestables ("C'est ce que montre l'Eurobaromètre »). Sur le plan qualitatif, l'argument est présenté de telle manière que la majorité apparaît s'accorder sur un point démocratique, fondamental et non controversé ('l'égalité entre femmes et hommes est un droit fondamental, l'égalité est un droit et doit devenir une priorité si elle n'est pas respectée'), proposition acceptable et qui relève donc de la volonté collective. Il en va de même pour le deuxième argument (1.1b), dont la formulation, plus réaliste, s'en rapporte à une «large majorité de citoyens». En invoquant un nombre important de personnes, et à deux reprises, l'argument est renforcé par la répétition de différentes sources, ne laissant ainsi aucune place au doute.

L'analyse de ces deux arguments où il est fait appel à la majorité montre qu'ils renforcent la proposition de faire de la question de l'inégalité entre genres une priorité. L'oratrice invite son auditoire à adopter son point de vue ('Nous devons faire une priorité du traitement des inégalités liées au genre') et à partager sa conviction profonde qu'agir ainsi contribuera à résoudre le problème de l'inégalité entre genres. Cette lecture est renforcée par l'emploi de la nécessité épistémique en (1).1b ( ( devrions »), associée à un agent collectif (« une large majorité de citoyens »).

21 Dans le même temps, la manière dont l'idée principale est renforcée indique que, pour éviter d'avoir à aller plus avant dans l'argumentation, Věra Jourová essaie de ne prendre à son compte aucun engagement à l'égard des propositions exprimées. Plutôt que d'avancer un argument qu'elle devrait étayer davantage, l'oratrice fait référence à ce que souhaite la majorité, et qui fonde la proposition de faire de l'inégalité entre les genres une priorité. Si, par la suite, cet argument explicite se révèle faux, il pourra n'être perçu que comme une simple erreur fondée sur des données erronées fournies par l'Eurobaromètre, ce qui aurait un effet infiniment moins menaçant pour la face de la locutrice. En ce sens, l'appel à la majorité atténue la force de l'argument tout en réduisant l'engagement de celle qui argumente à l'égard de l'acceptabilité de ce qui est affirmé. Ce faisant, la charge de la preuve ne revient pas à la politicienne, qui n'a donc 
pas à pousser plus loin l'argumentation. L'acceptabilité de la préconisation (1) est accrue par l'emploi d'un appel à la majorité dont les destinataires du discours font partie («pratiquement tous les citoyens européens», "une large majorité de citoyens »). Si l'auditoire mettait en doute la légitimité de son propos, il se contredirait lui-même (puisqu'il fait partie de la majorité des citoyens européens), et, plus grave encore, il nierait l'idée d'équité ("l'égalité des genres») et de démocratie («l'égalité entre femmes et hommes est un droit fondamental »), à laquelle il adhère pourtant.

Le deuxième exemple provient d'une interview politique du 15 mars 2009 lors de l'émission Politics Show de la BBC, émission au cours de laquelle le journaliste Jon Sopel interroge John Hutton, alors Secrétaire de la défense britannique. La discussion porte sur la participation de la Grande-Bretagne aux conflits irakien et afghan. Dans l'extrait présenté ici, Jon Sopel pose une question sur un incident au cours duquel le Royal Anglian Regiment de l'armée britannique est accueilli, à son retour, par un groupe de citoyens critiquant ses opérations armées ${ }^{6}$ :

Exemple (2) :

Jon Sopel :

Vous avez évoqué la nécessité de s'attaquer à l'extrémisme fondamentaliste et je ne peux m'empêcher de penser à ces images que nous avons vues au cours de la semaine à Luton, avec les soldats du Royal Anglian accueillis à leur retour par des banderoles et des slogans qui disaient «Allez au diable, les Royal Anglians, les bouchers de Basra ", je veux dire, qu'est-ce que vous en avez pensé quand vous avez vu ça?

John Hutton :

Je pense comme tout le monde dans notre pays : je me suis senti très, très choqué par les propos qui ont été tenus. Je pense qu'il faut juste garder à l'esprit qu'il n'y avait qu'une demi-douzaine de personnes au milieu d'une foule de plusieurs milliers de gens qui voulaient exprimer leur respect et leur gratitude à l'égard des soldats du Royal Anglian, de retour d'Irak. Je pense qu'il s'agissait d'une poignée de personnes qui étaient de toute évidence aux antipodes de ce qui se rapproche de l'opinion générale, démocratique, dans ce pays. Nous avons vu ce qu'elles avaient à dire et je pense que la plupart des gens dans le pays étaient écœurés par ce type d'allégation ridicule proférée à l'égard de nos hommes et femmes sous les drapeaux. [...] Jon Sopel :

Oui. En particulier, un député conservateur propose qu'il y ait une espèce de loi contre les incitations à la haine envers les personnels militaires en exercice. Seriezvous d'accord avec ça?

John Hutton :

Eh bien, je pense que le respect est la chose la plus importante dans ce débat. Je pense que les hommes et les femmes qui servent notre pays, qui portent notre uniforme ont droit au respect. Ils font un travail extraordinaire. Ils ont fait un travail extraordinaire en Irak, ils font un travail extraordinaire en Afghanistan. Et je pense que quand ils rentrent chez eux et voient des citoyens britanniques parlant, criant et se comportant de la sorte, je pense, je pense qu'il y a des leçons importantes à tirer pour nous tous face à cela. Le droit à la liberté d'expression est une composante très importante de notre mode de vie, mais j'ai la très ferme conviction que ces choses-là doivent être modérées. Nous... Il y a toujours cette idée dans la loi anglaise qu'on ne doit pas offenser inutilement les autres. Je suis convaincu que les lois dont nous disposons actuellement en matière d'ordre public sont assez complètes et permettent de traiter ce genre de problèmes à l'avenir.

Jon Sopel :

Peut-être que la police aurait dû traiter cette affaire autrement?

John Hutton : 
Je n'ai pas à faire de commentaires là-dessus, mais je pense que cela a laissé un goût très très amer à la majorité du grand public en Grande Bretagne et j'espère que ceux qui sont responsables de cette manifestation réfléchissent très sérieusement à ce qu'ils font.

Jon Sopel :

Et je sais que les soldats sont de solides gaillards, mais, je veux dire, est-ce qu'ils se sont sentis insultés?

John Hutton :

Je pense qu'il faudrait le leur demander, je pense que c'était probablement le cas en fait, oui. Pour ma part, ça m'a choqué, et je pense que c'est aussi vrai de la grande majorité de l'opinion publique britannique. Les gens ont des convictions très tranchées à l'égard de la guerre en Irak, ils ont des convictions tranchées concernant notre rôle en Afghanistan, mais je pense que ces convictions doivent s'exprimer sans porter contre les autres des allégations tout à fait ridicules et absurdes.

Dans cet extrait, John Hutton défend la thèse que la liberté d'expression doit être limitée en arguant du fait que la majorité des citoyens britanniques a été choquée par ce qui a été dit lorsque le régiment des Royal Anglians est revenu au pays, et en suggérant que les soldats eux-mêmes ont probablement été offensés par certaines remarques du public :

(1) (La liberté d'expression doit être limitée)

(1).1a La plupart des gens dans le pays ont été choqués par les slogans contre les Royal Anglians.

(1).1b Les soldats eux-mêmes ont été offensés par les allégations proférées à leur encontre.

Dans son argumentation, John Hutton propose que la liberté d'expression soit modérée en suggérant que sa proposition a des conséquences positives (réduire les offenses envers les autres). Malgré les conséquences positives potentielles de cette proposition, sa recommandation est sujette à controverse et la prétention de John Hutton à restreindre la liberté d'expression pourrait être taxée de mesure anti-démocratique. ${ }^{7}$ L'argumentation qu'il avance en soutien à cette proposition susceptible d'être contestée repose sur deux arguments qui, pris ensemble, renforcent la légitimité de ce qu'il préconise. Le premier argument ((1).1a), répété à quatre reprises pendant l'interview, fait référence à la majorité des citoyens britanniques (« tout le monde dans notre pays", "la plupart des gens dans le pays", "la majorité du grand public en Grande Bretagne », « la grande majorité de l'opinion publique britannique »). Le second argument ((1).1b) concerne les soldats qui étaient visés par les allégations.

Ce qui est remarquable, c'est que les deux arguments avancés pour défendre la ligne d'action prônée constituent tous deux des tentatives d'en masquer le caractère équivoque (cf. Bull 2008). Le politicien ne présente pas les arguments comme siens, mais les attribue à d'autres instances qui doivent être tenues pour responsables de leur contenu douteux ou incertain (la grande majorité et les soldats pensent que). Ces " procédés déresponsabilisants » (Holmes 1984: 360-361) sont employés pour atténuer la force illocutoire des arguments de John Hutton et attribuer la responsabilité de ces affirmations à un tiers. De toute évidence, les téléspectateurs regardant l'interview sont une composante de ce tiers (la grande majorité de l'opinion publique britannique) et ne peuvent facilement contester que les slogans insultants devraient être interdits, quand bien même cela impliquerait de restreindre la liberté d'expression. Ce faisant, John Hutton n'exprime aucun engagement personnel et manifeste clairement qu'il ne veut 
pas être tenu pour responsable de ce qu'il dit; il reporte ainsi sur d'autres la responsabilité de son acte de communication (cf. Bull, Fetzer \& Johansson 2008 : 326).

L'idée que John Hutton tente de réduire son engagement envers ses arguments est corroborée par l'emploi de l'indicateur d'attitude propositionnelle «Je pense », qu'il répète à plusieurs reprises dans la formulation de ses arguments pour signifier «Je suppose que ", «J'ai l'impression que» («je pense que la plupart des gens dans le pays ", « je pense que cela a laissé un goût très très amer à la majorité du grand public en Grande Bretagne ", "je pense que la grande majorité de l'opinion publique britannique »). Comme l'explique Berlin (2008 : 376), l'utilisation de Je pense constitue une ressource précieuse pour les politiciens habiles afin de manipuler la perception qu'ont les auditeurs de ce qui est communiqué tout en atténuant leur propre engagement quant à la vérité ou l'acceptabilité de la thèse avancée. Van Eemeren, Houtlosser et Snoeck Henkemans (2007 : 29) soulignent que l'emploi de cet indicateur d'attitude propositionnelle ne se limite pas à communiquer que le locuteur croit en quelque chose ; il suggère aussi que le locuteur considère « que le destinataire a besoin de cette information pour comprendre que la proposition avancée reflète une impression (subjective) du locuteur ». Ces auteurs montrent de manière convaincante que l'emploi de ces indicateurs «signifie que le locuteur anticipe les doutes du destinataire quant à l'acceptabilité de ce qu'il avance" et s'efforce de contrer ces doutes éventuels. En introduisant ses arguments par « je pense », John Hutton cherche à détourner le destinataire de l'idée qu'il disposerait de preuves solides de ce qu'il dit. L'auditoire qui accepte ses arguments, et implicitement le point de vue sujet à controverse, porte seul la responsabilité d'inférer des propos du politicien que ce qu'il avance est étayé par des preuves.

La répétition, dans cet extrait, des références à la volonté et aux préférences de la majorité (quatre fois) ainsi que de l'indicateur d'attitude propositionnelle « je pense » (trois fois) renforce mon analyse, selon laquelle John Hutton tente de suggérer qu'il n'assume aucune charge de la preuve pour les arguments qu'il avance. A coup de répétitions, il oriente l'attention de l'auditoire sur le fond de ses arguments (cf. van Rees 1996). De la sorte, Hutton suggère qu'il anticipe sur une possible mise en doute du fait que l'auditoire partage réellement les sentiments qu'il lui attribue. Pour éviter une telle critique, John Hutton répète à l'envi les mêmes arguments, pour indiquer qu'il maintient sa position et les raisons qui la justifient, que l'auditoire ne peut contester sans renier ses propres convictions.

\section{Résultats, enjeux et orientations futures}

Le présent article apporte un éclairage sur l'étude de l'appel à la majorité comme procédé argumentatif avancé en soutien d'une préconisation considérée comme acceptable au regard de ses conséquences. Contrairement aux analyses existantes de ce type d'argument, qui s'intéressent à la façon dont de tels appels étayent un point de vue descriptif, je me concentre sur les fonctions que jouent les appels à la majorité dans des débats politiques visant à asseoir l'acceptabilité d'une ligne d'action (par exemple, faire de l'inégalité entre les genres une priorité, ou restreindre la liberté d'expression).

Dans la présente analyse, les appels à la majorité ont été définis comme un procédé argumentatif étayant l'acceptabilité d'une affirmation par l'invocation des désirs, préférences et valeurs d'un grand nombre d'individus. Une telle définition permet de 
ne pas poser d'emblée le caractère fallacieux des appels à la majorité, pourtant affirmé par la plupart des auteurs qui ont traité cette forme d'argumentation, et de rendre ainsi justice aux débatteurs en ne présupposant plus qu'ils tentent de manipuler l'auditoire. Sur la base de cette définition, deux exemples ont été analysés en détail pour déterminer quels sont les engagements que les politiciens assument lorsqu'ils font appel à la majorité. L'étude a montré que cette forme d'argumentation est employée pour alléger la charge de la preuve du politicien vis-à-vis du point de vue qu'il avance. De la sorte, il cherche à éviter ou minimiser la critique, ce qui lui facilite considérablement la tâche au cours du débat. Le politicien présente ses opinions comme étant celles de la majorité, et suggère ainsi qu'il n'aurait pas à les défendre si elles se trouvaient contestées. Il donne le sentiment qu'il ne fait que rendre compte des opinions de la majorité et, partant, qu'il ne peut en être tenu pour responsable.

Ces observations appellent deux développements. En premier lieu, se pose la question du caractère fallacieux des appels à la majorité analysés dans les deux extraits de débats, et de la justification de la réponse à cette question. A première vue, il semble que nier tout engagement du locuteur à l'égard de ce qu'il avance est contradictoire avec les engagements institutionnels attachés au type d'activité dont relèvent ces échanges. Dans les interviews politiques et les débats parlementaires (les deux types d'activité en question), les politiciens sont censés défendre leurs positions et les étayer argumentativement ; s'ils ne se conforment pas à ces attentes, ils donnent le sentiment de ne pas assumer correctement leur rôle politique. Dans le même temps, le fait de chercher à éviter la critique lorsque l'on sait qu'on ne dispose pas de bons arguments en faveur de ce que l'on avance n'est pas fallacieux en soi.

La deuxième question à laquelle il s'agit de répondre est de déterminer dans quelle mesure réduire son engagement en faisant appel à la majorité constitue un mode d'action stratégique. Cela suppose de résoudre la contradiction suivante : d'un côté, les appels à la majorité peuvent être vus comme des tentatives d'écarter toute accusation de comportement antidémocratique. De l'autre, on l'a montré, les appels à la majorité cherchent à alléger la charge de la preuve pour le locuteur, ou à la faire peser sur les épaules de la majorité, qui peut avoir tort, le politicien quant à lui n'en étant pas comptable.

Les futurs travaux de recherche sur les appels à la majorité doivent se saisir de ces questionnements, et proposer une solution pour les problèmes qui se posent encore. En premier lieu, les recherches qualitatives de la même nature que celles présentées ici doivent être complétées par des recherches quantitatives, qui devraient permettre de tirer des conclusions généralisables sur la qualité de cette forme d'argumentation. Deuxièmement, il est nécessaire de poursuivre la recherche qualitative en étudiant un corpus d'exemples représentatifs permettant d'étudier des formes d'appels à la majorité plus variées et plus complexes que celles examinées ici. Troisièmement, il faut que soient définis des critères précis susceptibles d'être appliqués pour déterminer le caractère fallacieux des appels à la majorité, et distinguer ainsi systématiquement et de manière fiable entre cas raisonnables et cas déraisonnables. 


\section{BIBLIOGRAPHY}

Anderson, Charles. 1979. « The Place of Principles in Policy Analysis », The American Political Science Review $73: 3,711-723$

Andone, Corina. 2015. « Pragmatic Argumentation in European Practices of Political Accountability ", Argumentation $29: 1,1-18$

Berlin, Laurence N. 2008. " "I Think, Therefore..." Commitment in Political Testimony ", Journal of Language and Social Psychology $27: 4,372-383$

Brabanter, Philippe de \& Patrick Dendale. 2008. «Commitment : the Term and the Notions », Belgian Journal of Linguistics 22, 1-14

Brandom, Robert B. 1994. Making It Explicit : Reasoning, Representing, and Discursive Commitment (Cambridge, MA : Harvard University Press)

Bull, P. 2008. « "Slipperiness, Evasion, and Ambiguity.” Equivocation and Facework in Noncommittal Political Discourse », Journal of Language and Social Psychology 27 : 4, 333-344

Bull, Peter, Anita Fetzer \& Marjut Johansson. 2008. « Analysing the Fine Details of Political Commitment », Journal of Language and Social Psychology 27 : 4, 324-332

Cook, Fay Lomax, Jason Barabas \& Benjamin I. Page. 2002. « Invoking Public Opinion : Policy Elites and Social Security », Public Opinion Quarterly $66: 2,235-64$

Eemeren, Frans H. van \& Rob Grootendorst. 1984. Speech Acts in Argumentative Discussions. A Theoretical Model for the Analysis of Discussions Directed towards Solving Conflicts of Opinion (Dordrecht : Foris Publications)

Eemeren, Frans H., Peter Houtlosser \& A. Francisca Snoeck Henkemans. 2007. Argumentative Indicators in Discourse. A Pragma-Dialectical Study (Dordrecht : Springer)

Eemeren, Frans H. van \& Peter Houtlosser. 2008. « Rhetoric in a Dialectical Framework : Fallacies as Derailments of Strategic Maneuvering ", Edda Weigand (éd.) Dialogue and Rhetoric (Amsterdam \& Philadelphia : John Benjamins), 133-152

Godden, David M. 2008. « On Common Knowledge and Ad Populum : Acceptance as Grounds for Acceptability », Philosophy and Rhetoric $41: 2,101-129$

Govier, Trudy. 2005. A Practical Study of Argument (Toronto : Thomson \& Wadsworth)

Green, Mitchell. 2007. « Speech Acts ». Stanford Encyclopedia of Philosophy [En ligne : http:// plato.stanford.edu/entries/speech-acts]

Holmes, Janet. 1984. « Modifying Illocutionary Force », Journal of Pragmatics 8 :3; 345-365

Holzinger, Katharina, Janine Reinhard \& Jan Biesenbender. 2014. « Do Arguments Matter? Argumentation and Negotiation Success at the 1997 Amsterdam Intergovernmental Conference », European Political Science Review 6 :2, 283-307

Maillat, Didier. 2013. « Constraining Context Selection : On the Pragmatic Inevitability of Manipulation », Journal of Pragmatics 59, 190-199

Minot, Walter. 1981. «A rhetorical view of fallacies : Ad hominem and ad populum ». Rhetoric Society Quarterly $11: 4,222-235$ 
Rees, M. Agnes van. 1996. « Functions of repetition in informal discussions », Carla Bazzanella (éd.), Repetition in Dialogue (Tübingen : Max Niemeyer Verlag), 141-155

Searle, John. 1969. Speech Acts. An Essay in the Philosophy of Language (Cambridge : CUP)

Toulmin, Stephen, Richard D. Rieke \& Allan Janik. 1984. An Introduction to Reasoning (University of California : Macmillan)

Walton, Douglas. 1992. The Place of Emotion in Argument (University Park : Pennsylvania State University Press)

Walton, Douglas. 1999. Appeal to Popular Opinion (University Park : Pennsylvania State University Press)

\section{NOTES}

1. Pour Walton (1992: 65), «non seulement la variante de l'appel au sentiment ou à l'opinion populaire associée à l'argumentum ad populum traditionnel peut ne pas être fallacieuse dans certains contextes de discussion, mais elle peut même constituer une technique légitime et contribuer crucialement à la production d'une argumentation réussie ».

2. Walton affirme que cette reconstruction montre clairement que l'argumentation s'appuyant sur l'opinion populaire est une forme raisonnable d'argumentation.

3. Ce qui est déconcertant, c'est que Walton (1999) associe aussi les appels à l'opinion populaire à d'autres types d'arguments, comme l'appel à l'opinion d'expert. Cette approche rend pratiquement impossible de comprendre à quoi correspond exactement cette forme argumentative dans sa propre approche dialectique.

4. Godden (2008: 102) observe que "généralement, le fait qu'une affirmation appartient au savoir commun est considéré comme une raison de l'accepter, alors que les appels à l'opinion populaire sont considérés comme des tentatives fallacieuses de soutenir une affirmation. »

5. Les points de vue et arguments implicites sont représentés entre parenthèses.

6. Pour l'objectif de cet article, l'entretien est transcrit sans suivre les conventions de transcription, la prosodie et les autres phénomènes conversationnels n'intervenant pas dans mon analyse.

7. John Hutton rappelle à l'auditoire que les lois britanniques prescrivent clairement que l'on «n'offense pas inutilement les autres ». Malgré cet alibi juridique, sa suggestion de restreindre la liberté d'expression pourrait être apparaître antidémocratique, en particulier par les manifestants protestant contre les militaires britanniques, qui veulent exprimer librement leurs opinions, en particulier parce qu'ils ont des «convictions tranchées », comme l'explique John Hutton. C'est parce qu'il a anticipé sur le fait que ces opinions pouvaient être sujettes à controverse qu'il lui est nécessaire d'avancer des arguments en appui à sa position. Parce qu'il prévoit de possibles critiques, il argumente d'emblée ses opinions.

\section{ABSTRACTS}

The aim of this paper is to examine the various degrees of commitment of appeals to the majority as employed by politicians and their effects on the outcome of the discussion. The focus lies on 
indicators and expressions which tone down the force with which something is asserted. Close attention will be paid to propositional attitude indicators (such as I think [this is what the majority wants from us], I have the impression that [this is what people want]) which are commonly employed by politicians in such a way that only a weak or no burden of proof is at issue. In this endeavour, examples from political interviews and parliamentary discussions will serve as empirical material.

Cet article vise à examiner les différents degrés d'engagement des appels à la majorité qui sont employés par des politiciens, ainsi que leurs effets sur l'issue du débat. Il identifie les indicateurs et expressions qui atténuent la force avec laquelle quelque chose est affirmé. Une attention particulière sera prêtée aux indicateurs d'attitude propositionnelle (comme je pense [que c'est ce que la majorité attend de nous], j'ai l'impression [que c'est ce que les gens veulent]) communément employés par les politiciens de manière à n'assumer qu'une charge de la preuve légère, voire pas de charge de la preuve du tout. Dans ce but, des exemples issus d'interviews politiques et de débats parlementaires serviront de matériel empirique.

\section{INDEX}

Keywords: appeals to the majority, burden of proof, commitment, parliamentary debates, political interviews

Mots-clés: appels à la majorité, charge de la preuve, débats parlementaires, engagement, interview politique

\section{AUTHORS}

\section{CORINA ANDONE}

University of Amsterdam 